\title{
Investigation of deep levels in bulk GaN material grown by halide vapor phase epitaxy
}

\author{
Thien Duc Tran, Galia Pozina, Erik Janzén and Carl Hemmingsson
}

\section{Linköping University Post Print}

\section{Tweet}

N.B.: When citing this work, cite the original article.

Original Publication:

Thien Duc Tran, Galia Pozina, Erik Janzén and Carl Hemmingsson, Investigation of deep levels in bulk GaN material grown by halide vapor phase epitaxy, 2013, Journal of Applied Physics, (114), 15.

http://dx.doi.org/10.1063/1.4825052

Copyright: American Institute of Physics (AIP) http://www.aip.org/

Postprint available at: Linköping University Electronic Press http://urn.kb.se/resolve?urn=urn:nbn:se:liu:diva-102085 


\title{
Investigation of deep levels in bulk GaN material grown by halide vapor phase epitaxy
}

\author{
Tran Thien Duc, Galia Pozina, Erik Janzén, and Carl Hemmingsson \\ Department of Physics, Chemistry and Biology (IFM), Linköping University, S-581 83 Linköping, Sweden
}

(Received 2 July 2013; accepted 27 September 2013; published online 15 October 2013)

\begin{abstract}
Electron traps in thick free standing GaN grown by halide vapor phase epitaxy were characterized by deep level transient spectroscopy. The measurements revealed six electron traps with activation energy of 0.252 (E1), 0.53 (E2), 0.65 (E4), 0.69 (E3), 1.40 (E5), and 1.55 eV (E6), respectively. Among the observed levels, trap E6 has not been previously reported. The filling pulse method was employed to determine the temperature dependence of the capture cross section and to distinguish between point defects and extended defects. From these measurements, we have determined the capture cross section for level E1, E2, and E4 to $3.2 \times 10^{-16} \mathrm{~cm}^{2}, 2.2 \times 10^{-17} \mathrm{~cm}^{2}$, and $1.9 \times 10^{-17} \mathrm{~cm}^{2}$, respectively. All of the measured capture cross sections were temperature independent in the measured temperature range. From the electron capturing kinetic, we conclude that trap E1, E2, and E3 are associated with point defects. From the defect concentration profile obtained by double correlated deep level transient spectroscopy, we suggest that trap E4 and E6 are introduced by the polishing process. (C) 2013 AIP Publishing LLC. [http://dx.doi.org/10.1063/1.4825052]
\end{abstract}

\section{INTRODUCTION}

There has been a strong development of GaN and IIInitride compounds during the last two decades, and today these materials are widely used. ${ }^{1-4}$ This is due to the IIInitride properties such as a direct band gap, which can be varied from $0.7 \mathrm{eV}$ for InN through $3.4 \mathrm{eV}$ for $\mathrm{GaN}$ to $6.2 \mathrm{eV}$ for AlN, high breakdown voltage, and high electron mobility. These properties make the III-nitrides interesting for applications such as optoelectronic devices operating from infrared to deep $\mathrm{UV}^{4}$ as well as for high-power and high-temperature electronics. ${ }^{1-3}$ In order to improve the efficiency of these GaN based devices we need freestanding $\mathrm{GaN}$ substrates with a low impurity and defect concentration to avoid diffusion of impurities into the active layer and trapping of carriers injected from the active layer into the substrate. Additionally, to understand how these traps influence the performance of the devices, we have to understand the nonradiative recombination and trapping mechanism of carriers, and, therefore, origin and properties of defects in GaN must be investigated in detail. Deep-level transient spectroscopy (DLTS) is a useful tool to study deep levels in films or bulk. From the measurement, important parameters such as defect concentration, activation energy, and capture cross section can be determined.

There have been many previous studies of deep levels in $\mathrm{GaN}$ grown by various techniques, and, so far, most of the defect levels have been found when carrying out DLTS measurement in the temperature range of $80 \mathrm{~K}-500 \mathrm{~K} .^{5-10}$ There have been reports on measurements at higher temperatures, ${ }^{11-15}$ and among these the highest temperature at which DLTS peaks have been reported is about $620 \mathrm{~K} .{ }^{15}$ However, since energy levels of efficient recombination centers are situated in the middle of the band gap, and $\mathrm{GaN}$ is a wide-band-gap material, DLTS scans at higher temperatures are necessary. Despite that a large number of DLTS investigations have been reported in the temperature range $80 \mathrm{~K}-500 \mathrm{~K}$, the identities of most of the observed peaks are still speculative.
In this paper, we report results from a detailed DLTS study on thick freestanding bulk GaN grown by halide (or hydride) vapor phase epitaxy (HVPE). By extending the DLTS temperature scanning range up to $700 \mathrm{~K}$, we have been able to study deep electron traps with high activation energies. By using thick GaN with low threading dislocation densities (TDD), deep levels associated with structural defects should be of low concentration.

\section{EXPERIMENT}

The GaN layers were grown on a low-temperaturegrown buffer layer using a vertical HVPE system, for additional details of the growth system (see Ref. 16). The growth was performed under excess ammonia condition at a temperature of $1000{ }^{\circ} \mathrm{C}$ and a total pressure of $1 \mathrm{~atm}$. A mixture of $\mathrm{H}_{2}$ and $\mathrm{N}_{2}$ with a ratio of 5:2 was used as carrier gas for the precursors $\mathrm{NH}_{3}$ and $\mathrm{GaCl}$. By using a mixture of $\mathrm{H}_{2}$ and $\mathrm{N}_{2}$, we obtain laminar flow and growth conditions that prevent parasitic deposition of $\mathrm{GaN}$ in the inlet. For additional details about the growth process, see Ref. 17. The as-grown surface of thick HVPE grown GaN is rough with large hillocks and ridges. Thus, in order to obtain a smooth surface for making contacts, the layers were mechanically polished down to a thickness of $\sim 0.5 \mathrm{~mm}$. The final polishing step was performed with $1 \mu \mathrm{m}$ diamond slurry. By using room-temperature cathodoluminescence (CL) spectroscopy, the TDD was estimated from the dark spot density to be about $5 \times 10^{6} \mathrm{~cm}^{-2}$ (for details about the technique, see Ref. 18). For the CL measurements, we were using a Leo 1500 Gemini scanning electron microscope equipped with a MonoCL system from Oxford Instruments using a 1200 lines $/ \mathrm{mm}$ grating blazed at $500 \mathrm{~nm}$. The diode structure was fabricated by vacuum evaporation using $\mathrm{Au} / \mathrm{Ni}(80 / 40 \mathrm{~nm})$ as Schottky contacts and Ti/Al $(40 / 150 \mathrm{~nm})$ as Ohmic contacts. First, the samples were chemically cleaned, and then the Ohmic contacts with a diameter of $3 \mathrm{~mm}$ were formed. 
The contacts were annealed at $550^{\circ} \mathrm{C}$ in $\mathrm{Ar}$ gas for $5 \mathrm{~min}$ to enhance Ohmic contact formation. ${ }^{19}$ Finally, Schottky contacts with a diameter of $1.2 \mathrm{~mm}$ were fabricated. From the current voltage measurement (IV), the diodes showed a high rectifying characteristic with a leakage current of $2 \mathrm{nA}$ at a reverse bias of $-10 \mathrm{~V}$ at room temperature. By capacitance voltage measurements $(\mathrm{CV})$, the net donor concentration at room temperature was determined to be approximately $1.8 \times 10^{16} \mathrm{~cm}^{-3}$. In order to determine the temperature dependence of the free electron concentration, Hall measurements were performed in a homemade setup. The magnetic field was $0.85 \mathrm{~T}$. For the Hall measurement, Ohmic silver contacts were evaporated in the Van der Pauw geometry. In order to avoid the influence of the highly conductive layer close to the sapphire substrate the backside of the layers $\sim 200 \mu \mathrm{m}$ were polished down before the Hall measurements. ${ }^{20}$ The DLTS measurements were performed using the following parameters: a temperature range of $80-700 \mathrm{~K}$, a filling pulse width range of $50-10 \mathrm{~ms}$, a steady reverse bias of $-10 \mathrm{~V}$, and the filling pulse heights of $6.5 \mathrm{~V}, 9.5 \mathrm{~V}$, and $10 \mathrm{~V}$. The DLTS data were investigated by using double box-car simulation ${ }^{21}$ to yield DLTS spectra at different rate windows from which the activation energy and the interception capture cross section were extracted. The capture cross section was measured more accurately by using the filling pulse method in which the amplitude of DLTS peak was determined as a function of the filling pulse widths from $50 \mathrm{~ns}$ to $700 \mathrm{~ns}$.

In the case of point defects, the concentration of the filled level is considered as the function of the filling pulse width

$$
N_{T}\left(t_{p}\right)=N_{T}\left[1-e^{-\left(\sigma_{n} n v_{t h} t_{p}\right)}\right],
$$

where $\mathrm{N}_{\mathrm{T}}$ is the concentration of level, $n$ is the free carrier concentration, and $v_{t h}$ is the average thermal velocity of the majority carriers. Since the amplitude of the DLTS peak is proportional to the concentration of the filled trap level, the amplitude of the DLTS peak $\Delta S_{\text {peak }}$ as a function of filling pulse width $t_{p}$ can be written

$$
\Delta S_{\text {peak }}\left(t_{p}\right)=\Delta S_{\text {peak }}\left(t_{p} \rightarrow \infty\right)\left[1-e^{-\left(\sigma_{n} n v_{t h} t_{p}\right)}\right],
$$

where $\Delta S_{\text {peak }}\left(t_{p} \rightarrow \infty\right)$ is the peak amplitude when $t_{p}$ is sufficient for filling all traps by majority carriers (electrons). If the contribution of the free carrier tail into the depletion region is taken into account, Eq. (2) has to be modified 22,23

$$
\Delta S_{\text {peak }}\left(t_{p}\right)=\alpha\left[1-e^{-\left(\sigma_{n} n v_{t h} t_{p}\right)}\right]+\beta \ln \left(\gamma t_{p}\right) .
$$

Here is the fitting parameter $\alpha$ related to the concentration of traps and the additional logarithmic term including the fitting parameters $\beta$ and $\gamma$ accounts for electron capture in the free carrier Debye-tail. By fitting Eq. (3) to the experimental data, the capture cross section $\sigma_{\mathrm{n}}$ can be determined.

For an extended defect, the amplitude of the DLTS peak is proportional to the filling pulse width as the following function: ${ }^{23,24}$

$$
\Delta S_{\text {peak }}\left(t_{p}\right)=\delta \ln \left(t_{p}\right),
$$

where $\delta$ is a constant determined by fitting the experimental data. This dependence of the peak amplitude is proposed by Wosinśsi ${ }^{24}$ based on earlier reports. In this model, the Coulombic barrier height caused by the dislocation core increases as the number of electrons gets trapped. This limits the electron capture rate and makes the DLTS signal of the dislocation linearly dependent on the logarithm of $t_{p}$. Therefore, the filling pulse width method is an effective and simple way to distinguish point defects from extended defects. Other different features between the two types of defects are discussed more in detail in Ref. 25.

\section{RESULT AND DISCUSSION}

The DLTS spectra using different pulse heights of $6.5 \mathrm{~V}$ (a) and $9.5 \mathrm{~V} \mathrm{(b)} \mathrm{with} \mathrm{a} \mathrm{reverse} \mathrm{bias} \mathrm{of}-10 \mathrm{~V}$ and a rate window of $10 / 20 \mathrm{~ms}$, corresponding to the emission rate of $69.3 \mathrm{~s}^{-1}$, are shown in Fig. 1. The energy position and an estimation of the capture cross section of the traps were determined by plotting the thermal emission rates (divided by $\mathrm{T}^{2}$ taking into account also the temperature dependencies of the thermal velocity and the density of states in the conduction band) as a function of 1000/T (so called Arrhenius plot) (see Fig. 2). From the amplitudes of the DLTS peaks, using the equation in Ref. 21, the trap concentrations are obtained. It is worth mentioning that the trap concentrations were determined, considering the effect of the free electron carrier tail $\lambda$ (the so-called Debye tail). This correction is especially important for deep levels in wide bandgap semiconductors since the energy levels can be very deep, and, consequently, the free carrier tail can extend deep into the space charge region. The obtained trap parameters are given in details in Table I.

The DLTS spectra shown in Fig. 1(a) reveals six peaks labeled E1, E2, E3, E4, E5, and E6, respectively. When increasing the pulse height up to $9.5 \mathrm{~V}$, the E3 and E5 peaks are completely immersed by the dominating E4 peak. The traps E1-E5 are typically found in $\mathrm{GaN}$ and have been evaluated previously.

The level E1 $\left(\mathrm{E}_{\mathrm{c}}-0.252 \mathrm{eV}\right)$ and the level E2 $\left(\mathrm{E}_{\mathrm{c}}\right.$ $-0.53 \mathrm{eV}$ ) are commonly observed in $\mathrm{GaN}$ grown by

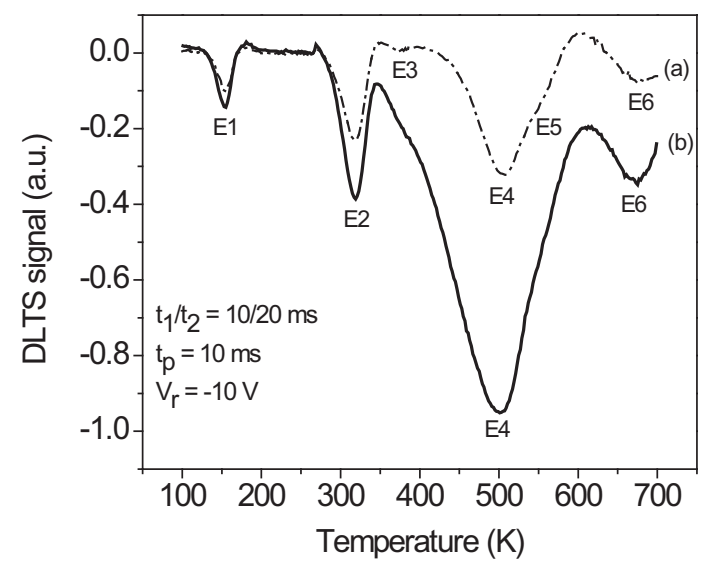

FIG. 1. DLTS spectra of thick freestanding GaN layer with $\mathrm{t}_{1} / \mathrm{t}_{2}=10 / 20 \mathrm{~ms}$, a reverse bias of $-10 \mathrm{~V}$, and a filling pulse width of $10 \mathrm{~ms}$ : (a) with a pulse height of $6.5 \mathrm{~V}$; (b) with a pulse height of $9.5 \mathrm{~V}$. 


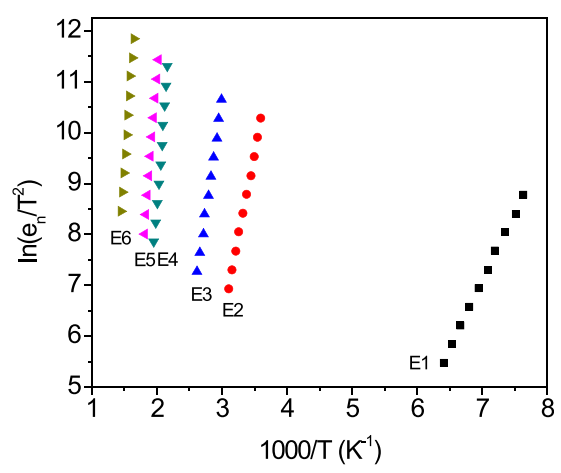

FIG. 2. Arrhenius plots of different deep levels observed by DLTS.

MOCVD,${ }^{12}$ HVPE, ${ }^{5}$ MOVPE,${ }^{23}$ and MBE. ${ }^{13}$ Thus, the existence of these traps seems to be independent of growth technique. The trap E1 has been reported to have an electron capture process behavior like a line defect, ${ }^{9,23}$ and it has been suggested to be related to native defects such as $\mathrm{O}_{\mathrm{N}}$ (Ref. 26) that are strongly bonded along threading dislocation core site. However, in studies by Ito et al. ${ }^{11}$ on GaN layers grown on AlN and sapphire it has been observed that the concentration of E1 does not depend on the TDD. Additionally, it has been suggested by Fang et al. ${ }^{8}$ that trap E1 (labeled D in Ref. 8) is related to a defect complex involving $\mathrm{V}_{\mathrm{Ga}}$ such as $\mathrm{V}_{\mathrm{N}}-\mathrm{V}_{\mathrm{Ga}}$.

One of the stronger peaks in the spectra is E2. This level has been reported by several authors, and in many reports it has been associated with the nitrogen antisite $\left(\mathrm{N}_{\mathrm{Ga}}\right){ }^{5,6,9,27}$ More recently, Look et al. ${ }^{10}$ performed measurements before and after $1 \mathrm{MeV}$ electron irradiation of HVPE grown GaN sample, and they did not observe any changes in concentration for this trap. Considering this and that it is an electron trap relatively close to the conduction band, they suggested that this level is associated with some more complex defect involving a common impurity such as $\mathrm{Si}, \mathrm{O}$, or $\mathrm{C}$ or possibly $\mathrm{C}_{\mathrm{Ga}}$. However, there have been other investigations on the impact of carbon incorporation in n-type $\mathrm{GaN}^{28,29}$ In Ref. 28, Armstrong et al. found that the concentration of the trap E2 was more or less unchanged by carbon intentionally introduced during the growth. They conclude that this deep level is not related to carbon, which should be due to an intrinsic defect. The conclusion is in agreement with our observation since in HVPE, there is no source of carbon, so the presence of $\mathrm{C}_{\mathrm{Ga}}$ can be excluded.

TABLE I. The energy position $E_{t}$, trap concentration $N_{t}$ using a filling pulse amplitude of $9.5 \mathrm{~V}$ and $6.5 \mathrm{~V}$, respectively, capture cross section $\sigma_{\text {int }}$ calculated from the Arrhenius plot and capture cross section $\sigma_{\text {meas }}$ determined by the filling pulse method.

\begin{tabular}{lccccc}
\hline \hline $\begin{array}{l}\text { Trap } \\
\text { level }\end{array}$ & $\mathrm{E}_{\mathrm{c}}$-Et $(\mathrm{eV})$ & $\begin{array}{c}\mathrm{N}_{\mathrm{t}}\left(\mathrm{cm}^{-3}\right) \\
\left(\mathrm{V}_{\mathrm{p}}=9.5 \mathrm{~V}\right)\end{array}$ & $\begin{array}{c}\mathrm{N}_{\mathrm{t}}\left(\mathrm{cm}^{-3}\right) \\
\left(\mathrm{V}_{\mathrm{p}}=6.5 \mathrm{~V}\right)\end{array}$ & $\sigma_{\text {int }}\left(\mathrm{cm}^{2}\right)$ & $\sigma_{\text {meas }}\left(\mathrm{cm}^{2}\right)$ \\
\hline E1 & $0.252 \pm 0.003$ & $3.9 \times 10^{13}$ & $3.5 \times 10^{13}$ & $9.6 \times 10^{-16}$ & $3.2 \times 10^{-16}$ \\
E2 & $0.53 \pm 0.01$ & $9.0 \times 10^{14}$ & $7.0 \times 10^{14}$ & $2.1 \times 10^{-16}$ & $2.2 \times 10^{-17}$ \\
E3 & $0.69 \pm 0.02$ & $\sim 10^{12}$ & $6.5 \times 10^{12}$ & $1.3 \times 10^{-15}$ & $\ldots$ \\
E4 & $0.65 \pm 0.05$ & $2.2 \times 10^{15}$ & $9.8 \times 10^{14}$ & $1.9 \times 10^{-18}$ & $1.9 \times 10^{-17}$ \\
E5 & $1.40 \pm 0.02$ & $\sim 10^{12}$ & $\sim 10^{12}$ & $3.0 \times 10^{-12}$ & $\ldots$ \\
E6 & $1.55 \pm 0.05$ & $1.2 \times 10^{15}$ & $2.9 \times 10^{14}$ & $2.9 \times 10^{-13}$ & $\cdots$ \\
\hline \hline
\end{tabular}

The signal from trap E3 is weak and almost completely immersed by the strong peak E4. However, despite this we were able to determine the activation energy to $\sim \mathrm{E}_{\mathrm{C}}$ $-0.69 \mathrm{eV}$ and the capture cross section to $1.3 \times 10^{-15} \mathrm{~cm}^{2}$.

The strongest peak E4 is commonly observed in GaN and has been reported previously. ${ }^{5,6}$ The nature of this defect is not clear, however, it has been suggested that it is linked to a nitrogen antisite. ${ }^{5,9}$ Since the concentration of defects associated with the peak E4 apparently increases when increasing the filling pulse amplitude (i.e., measuring closer to the surface), we suggest that trap E4 may be related to some interfacial defect such as stacking fault that is one of the most typical defects close to the boundary probably introduced during the mechanical polishing process.

Trap E6 $\left(\mathrm{E}_{\mathrm{C}}-1.55 \mathrm{eV}\right)$ has not previously been reported. To determine the exact activation energy of trap E6, it is necessary to perform DLTS scans at high temperatures. Due to temperature-range limitation of our system, the activation energy determined from the Arrhenius plot is uncertain but roughly $1.55 \pm 0.05 \mathrm{eV}$ which locates the level near the middle of the band gap. A deep level with an activation energy of $1.44 \pm 0.3 \mathrm{eV}$ has previously been reported by Lee et $_{\text {al. }}{ }^{14}$ (the large error range is related to limitations of high temperature DLTS measurements in their system). However, the DLTS peak position of the $1.44 \mathrm{eV}$ level is at lower temperatures (around $500 \mathrm{~K}$ ), and consequently the reported capture cross section estimated from the Arrhenius plot differs significantly to trap E6. Considering the large error range and the peak position of the trap reported by Lee et al., we suggest that the $1.44 \pm 0.3 \mathrm{eV}$ level corresponds to the E5 trap with an activation energy about $1.40 \mathrm{eV}$. Lee et al. studied MOCVD grown GaN layers using trimethylgallium or triethylgallium as the column III precursor and independently of precursor, trap E5 was detected. No suggestion of the origin of trap E5 was reported, but, unlike MOCVD, the HVPE process does not involve metalorganics, thus providing an environment without carbon. It means that we can rule out that trap E5 is related to carbon or carbon complexes. Since the concentration of trap E6 is much higher near the surface as judged from the increasing concentration of defects using a larger filling pulse amplitude (see Table I), we suggest that trap E6 is related to an interfacial defect such as a stacking fault or a dislocation that may have been introduced by the polishing process. However, the identities of both trap E5 and E6 is still an open question and need further investigations.

In order to verify that the concentration of trap E4 and E6 is higher closer to the surface, double correlated DLTS measurements (DDLTS) were performed. As can be seen in Fig. 3, the concentration of traps increases strongly as we are approaching the surface. It is known that polishing can introduce subsurface damages, and in HVPE grown GaN substrates there have been reported that even after chemical mechanical polishing (CMP) there is a damaged region within $1.48 \mu \mathrm{m}$ from the surface. ${ }^{30}$ This depth is within the measured depth, suggesting that both trap E4 and E6 are associated with defects introduced by the polishing process.

Figure 4 shows DLTS spectra of thick freestanding GaN layer using a rate window $t_{1} / t_{2}=10 / 20 \mathrm{~ms}$, a reverse bias of 


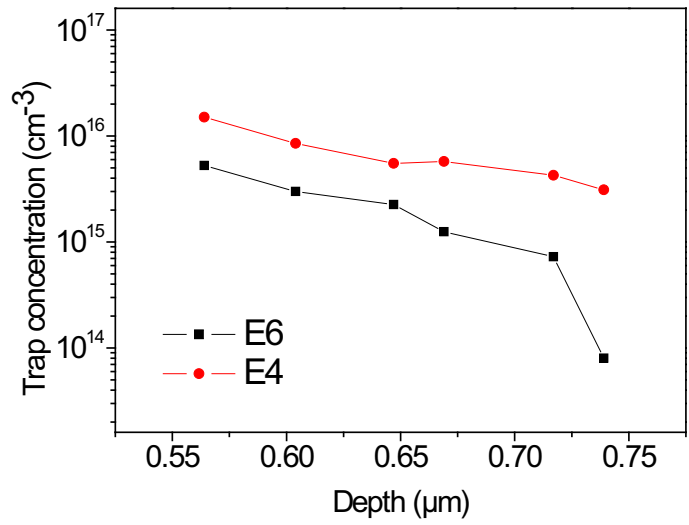

FIG. 3. Deep level profiles of trap E4 and trap E6 in the depth range $550-750 \mathrm{~nm}$ from the surface obtained by DDLTS.

$-10 \mathrm{~V}$, and a pulse height of $10 \mathrm{~V}$ using different filling pulse widths in the temperature range $100-600 \mathrm{~K}$. A decrease of the filling pulse duration makes the number of filled traps decrease, and thereby the amplitude of DLTS peaks also decreases. As can be seen, the amplitude of peaks E1, E2, E4, and E5 is decreasing. We observe that peak E5 is decreasing less than E4 which shows that the electron capture cross section for trap E5 is larger than E4. The DLTS peak amplitude of E1, E2, and E4 at 151, 319, and $465 \mathrm{~K}$ as a function of filling pulse width $t_{p}$ is shown in Fig. 4. Peak E5 was not possible to resolve due to the severe overlapping with peak E4. Excellent fitting to the experimental result was obtained by using Eq. (3), as depicted with the solid lines in Fig. 5, where we have taking into account the free carrier tail into the space charge region. Using a model assuming capturing to extended defects (Eq. (4)) resulted in a poor fit. Thus, it suggests that all the measured trap levels are associated with point defects. ${ }^{25}$ Assuming that the free carrier concentration at room temperature is approximately the donor concentration and using the free carrier concentration measured by Hall for the low temperature range from 100 to $160 \mathrm{~K}$, the capture cross section of each trap was obtained (see Table I).

In previous studies, Cho and co-workers have studied E1 and E2 by varying the filling pulse time in DLTS

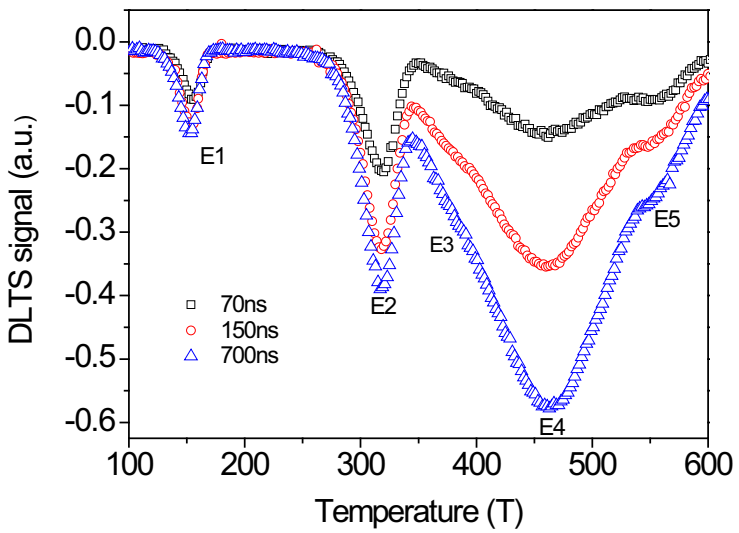

FIG. 4. DLTS spectra of thick freestanding GaN layer with $\mathrm{t}_{1} / \mathrm{t}_{2}=10 / 20 \mathrm{~ms}$, a reverse bias of $-10 \mathrm{~V}$, and pulse height of $10 \mathrm{~V}$ using filling pulses widths in the range $70-700 \mathrm{~ns}$.

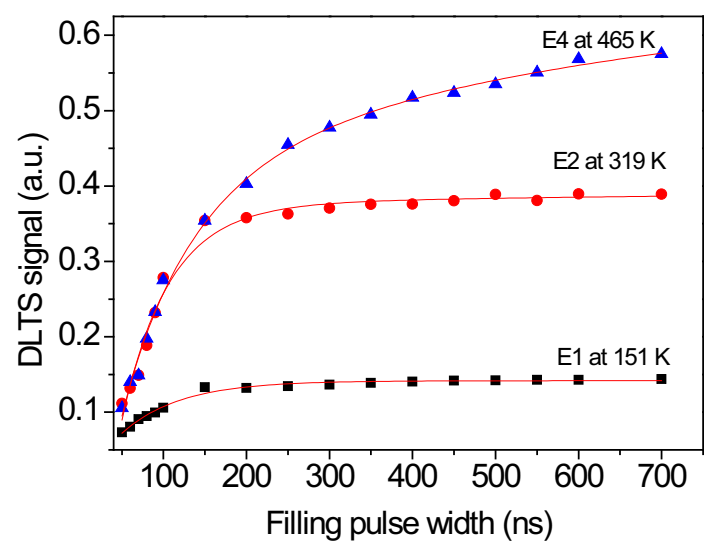

FIG. 5. The dependence of the DLTS signal of the E1, E2, E4 traps on the filling pulse width $t_{\mathrm{p}}$.

measurement. ${ }^{9}$ They concluded that E1 is related to a line defect with dangling bonds along the dislocation core site, and E2 is due to point defect which may be related to nitrogen antisites. Our measurements show that E1 is a point defect which contradicts their observation. However, one important difference between the studies is that they did the measurement on MOCVD grown $\mathrm{GaN}$ on sapphire with a high TDD while in this investigation we are using thick HVPE GaN with a TDD about 2 orders of magnitude less. Fang et al. ${ }^{8}$ did DLTS studies on thick GaN grown by HVPE with low TDD and GaN grown by MOCVD with high TDD and reactive molecular beam epitaxy. They concluded that many defects that behaved as line defects in samples with high TDD behaved as point defects in the sample with low TDD. The most likely explanation is that many electron traps tend to segregate around dislocations and, consequently, behave like a line defect in material with high TDD.

The amplitude of the DLTS peaks E1, E2, and E4 as a function of filling pulse width was extracted at different temperatures by using different rate windows to study the temperature dependence of the electron capture cross sections of the traps, as shown in Fig. 6. In the studied range, no temperature dependence of the capture cross sections is observed. This result is in good agreement with other studies. ${ }^{8,31}$

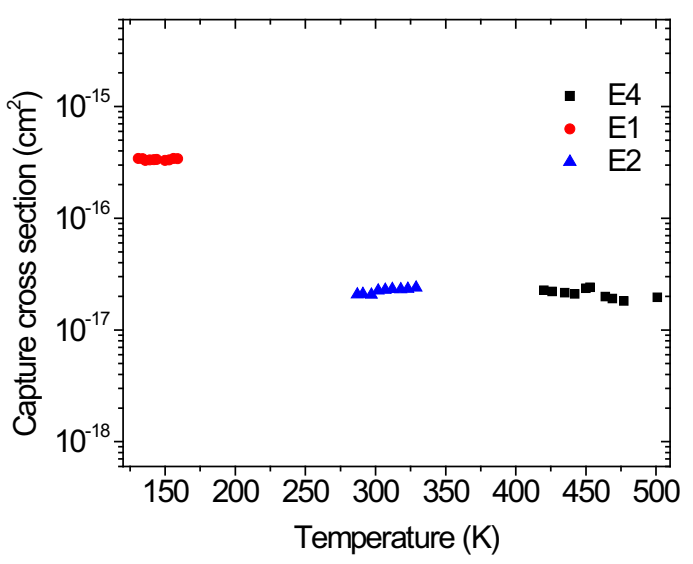

FIG. 6. The temperature dependence of the capture cross section of traps $\mathrm{E} 1, \mathrm{E} 2$, and $\mathrm{E} 4$. 


\section{CONCLUSION}

In our study, freestanding GaN bulk grown by HVPE has been electrically characterized by DLTS. Six traps labeled E1 $\left(\mathrm{E}_{\mathrm{C}}-0.252 \mathrm{eV}\right), \mathrm{E} 2\left(\mathrm{E}_{\mathrm{C}}-0.53 \mathrm{eV}\right), \mathrm{E} 3$ $\left(E_{C}-0.69 \mathrm{eV}\right), \mathrm{E} 4\left(\mathrm{E}_{\mathrm{C}}-0.65 \mathrm{eV}\right), \mathrm{E} 5\left(\mathrm{E}_{\mathrm{C}}-1.40 \mathrm{eV}\right)$, and E6 $\left(E_{C}-1.55 \mathrm{eV}\right)$ were observed. By DDLTS measurements, we observe that the concentration of trap E4 and E6 is increasing closer to the surface. Thus, we suggest that these two levels are associated with defects introduced during the mechanical polishing process. To determine the origin of these defects further investigations are necessary. The filling pulse method was used to measure the capture cross section, its temperature dependence, and to distinguish point defects from extended defects. From these measurements, we can conclude that trap E1, E2, and E4 are point defects. In the studied temperature range, no temperature dependence of the capture cross sections was observed.

\section{ACKNOWLEDGMENTS}

This work was supported by the Swedish Research Science Council (VR) and Swedish Energy Agency.

\footnotetext{
${ }^{1}$ A. Isamu, A. Hiroshi, S. Shigetoshi, S. Hiromitsu, T. Toshiyuki, and K. Masayoshi, Jpn. J. Appl. Phys. 34, 1517 (1995).

${ }^{2}$ Y. Arakawa, IEEE J. Sel. Top. Quantum Electron. 8, 823 (2002).

${ }^{3}$ Y. Dora, A. Chakraborty, L. Mccarthy, S. Keller, S. P. Denbaars, and U. K. Mishra, IEEE Electron Device Lett. 27, 713 (2006).

${ }^{4}$ S. Nakamura, T. Mukai, and M. Senod, Appl. Phys. Lett. 64, 1687 (1994).

${ }^{5}$ P. Hacke, T. Detchprohm, K. Hiramatsu, N. Sawaki, K. Tadatomo, and K. Miyake, J. Appl. Phys. 76, 304 (1994).

${ }^{6}$ D. Haase, M. Schmid, W. Kürner, A. Dörnen, V. Härle, and A. Do, Appl. Phys. Lett. 69, 2525 (1996).

${ }^{7}$ Z.-Q. Fang, D. C. Look, J. Jasinski, M. Benamara, Z. Liliental-Weber, and R. J. Molnar, Appl. Phys. Lett. 78, 332 (2001).
}

${ }^{8}$ Z.-Q. Fang, D. C. Look, and L. Polenta, J. Phys.: Condens. Matter 14, 13061 (2002).

${ }^{9}$ H. K. Cho, C. S. Kim, and C.-H. Hong, J. Appl. Phys. 94, 1485 (2003).

${ }^{10}$ D. C. Look, Z.-Q. Fang, and B. Claflin, J. Cryst. Growth 281, 143 (2005).

${ }^{11}$ T. Ito, M. Yoshikawa, A. Watanabe, and T. Egawa, Phys. Status Solidi C 5, 2998 (2008).

${ }^{12}$ Y. Tokuda, Y. Matsuoka, H. Ueda, O. Ishiguro, N. Soejima, and T. Kachi, Superlattices Microstruct. 40, 268 (2006).

${ }^{13}$ C. D. Wang, L. S. Yu, S. S. Lau, E. T. Yu, W. Kim, A. E. Botchkarev, and H. Morkoç, Appl. Phys. Lett. 72, 1211 (1998).

${ }^{14}$ W. I. Lee, T. C. Huang, J. D. Guo, and M. S. Feng, Appl. Phys. Lett. 67, 1721 (1995).

${ }^{15}$ D. Johnstone, S. Biyikli, S. Dogan, Y. T. Moon, F. Yun, and H. Morkoc, Proc. SPIE 5739, 7 (2005).

${ }^{16}$ C. Hemmingsson, P. P. Paskov, G. Pozina, M. Heuken, B. Schineller, and B. Monemar, Superlattices Microstruct. 40, 205 (2006).

${ }^{17}$ C. Hemmingsson, G. Pozina, M. Heuken, B. Schineller, and B. Monemar, J. Cryst. Growth 310, 906 (2008).

${ }^{18}$ S. J. Rosner, E. C. Carr, M. J. Ludowise, G. Girolami, and H. I. Erikson, Appl. Phys. Lett. 70, 420 (1997).

${ }^{19}$ B. P. Luther, S. E. Mohney, T. N. Jackson, M. A. Khan, Q. Chen, and J. W. Yang, Appl. Phys. Lett. 70, 57 (1997).

${ }^{20}$ W. Götz, J. Walker, L. T. Romano, N. M. Johnson, and R. J. Molnar, Mater. Res. Soc. Symp. Proc. 449, 525 (1997).

${ }^{21}$ D. V. Lang, J. Appl. Phys. 45, 3023 (1974).

${ }^{22}$ D. Pons, J. Appl. Phys. 55, 3644 (1984).

${ }^{23}$ E. PŁaczek-Popko, J. Trzmiel, E. Zielony, S. Grzanka, R. Czernecki, and T. Suski, Physica B 404, 4889 (2009).

${ }^{24}$ T. Wosiński, J. Appl. Phys. 65, 1566 (1989).

${ }^{25}$ Ł. Gelczuk, M. Dabrowska-Szata, and G. Jóźwiak, Mater. Sci. Poland 23, 625 (2005).

${ }^{26}$ C. B. Soh, S. J. Chua, H. F. Lim, D. Z. Chi, S. Tripathy, and W. Liu, J. Appl. Phys. 96, 1341 (2004).

${ }^{27}$ H. M. Chung, W. C. Chuang, Y. C. Pan, C. C. Tsai, M. C. Lee, W. H. Chen, W. K. Chen, C. I. Chiang, C. H. Lin, and H. Chang, Appl. Phys. Lett. 76, 897 (2000).

${ }^{28}$ A. Armstrong, A. R. Arehart, D. Green, U. K. Mishra, J. S. Speck, and S. A. Ringel, J. Appl. Phys. 98, 053704 (2005).

${ }^{29}$ C. H. Seager, A. F. Wright, J. Yu, and W. Goütz, J. Appl. Phys. 92, 6553 (2002).

${ }^{30}$ K. Y. Lai, M. A. L. Johnson, T. Paskova, A. D. Hanser, K. Udwary, E. A. Preble, and K. R. Evans, Phys. Status Solidi C 6, S325 (2009).

${ }^{31}$ J. Pernot and P. Muret, J. Appl. Phys. 103, 023704 (2008). 